\title{
Autosomal, sex and B chromosomes in Eyprepocnemis plorans (Orthoptera) viewed with restriction endonuclease in situ digestion
}

\author{
C. LÓPEZ-FERNÁNDEZ, R. MEZZANOTTE* \& J. GOSÁLVEZ \\ Unidad de Genética, Departamento de Biologia, C-XV. Facultad de Ciencias, Universidad Autónoma de Madrid 28049, \\ Madrid, Spain and */stituto di Biologia Generale, Facolta di Medicina e Chirurgia, via Ospedale 121.09124, Cagliari, Italia
}

Similarities and differences between different subsets of heterochromatin localized on the autosomes, sex chromosomes and a B-chromosome in the genome of the grasshopper Eyprepocnemis plorans were analysed by in-situ digestion with 23 different restriction endonucleases, three unspecific nucleases, C-banding and specific fluorescent DNA-ligands. Results show an equilocal distribution of the different heterochromatic subsets in this species and provide a possible evolutionary origin for the $\mathrm{B}$ chromosome.

Keywords: B-chromosomes, heterochromatin, insects cytogenetics, restriction endonucleases.

\section{Introduction}

B-chromosomes are a special class of chromatin in many species of animals and plants which show peculiar characteristic features. For example, (i) they are supernumerary to the main chromosome complement, (ii) they are often transmitted in a non-Mendelian fashion and (iii) they can affect the genetics of a species through their effects on meiotic recombination (see Jones \& Rees, 1982 for review). To date, however, the molecular mechanism(s) through which the B-chromosomes act on the individuals carrying them, as well as their organization in terms of DNA-protein structure, are not known. In particular, the origin of B-chromosomes, and their relationship with other components of the genome, is a difficult problem. In this connection, Hewitt (1979) stated that 'the relationship of B-chromosomes to the regular complement will be determined through molecular based studies such as nucleic acid hybridization and specific chromosome banding procedures'. Unfortunately, more than 10 years later the nature, function and origin of such 'illegal members of inheritance' still remain largely unknown, despite many different investigations.

The discovery of banding techniques allowed the differentiation of metaphase chromosomes into visible areas that correspond to specific chromosome domains. Different types of chromatin could thus be recognized depending on their reaction to a number of chemical, physical or enzymatic treatments (i.e. G-, R-, C-bands). Moreover, the improvement of such techniques permitted new detailed analyses and differentiation into discrete subclasses and blocks of chromatin. For instance, a positive homogeneous reaction to C-banding, usually identified as constitutive heterochromatin (Sumner, 1972), is now known not to represent the same type of material in a given organism (John et al., 1985; Gosálvez et al., 1987; LópezFernández et al., 1989). In this study we used a number of techniques including in-situ digestion with restriction endonucleases, to analyse the different types of chromatin present in the genome of the grasshopper Eyprepocnemis plorans. This species was selected in order to study the origin of an extra B-chromosome as well as its relationship with the different classes of chromatin in its karyotype. The results obtained lead us to hypothesize that the origin of this particular B-chromosome might involve genomic material which was part of an autosome. This material seems to have undergone rearrangements and followed an evolutionary pathway different from that of the autosomes.

\section{Materials and methods}

Males of Eyprepocnemis plorans collected in Sardinia (Italy) were used in this study. Testes were fixed in 3:1 
ethanol:acetic acid and some follicles were squashed in a drop of 45 per cent acetic acid. Coverslips were removed after immersing the preparations in liquid nitrogen. Fluorescence analysis was carried out using Chromomycin A3 (CMA3), Dystamycin (DA) and 4'-6 diamidine-phenyl indole (DAPI) according to the method developed by Schweizer $(1976 ; 1980)$.

Twenty-three restriction endonucleases (REs) (see Table 1) were used to digest fresh air-dried preparations. The slides were incubated with $100 \mu \mathrm{l}$ of incubation buffer plus 30 units of each enzyme. All REs, except Taq I, were incubated overnight at $37^{\circ} \mathrm{C}$. Incubation with Taq I was at $65^{\circ} \mathrm{C}$ overnight. The same times and temperatures were employed in control experiments using only the incubation buffer. Cytological preparations were stained with Giemsa 2 per cent in phosphate buffer ( $\mathrm{pH}$ 6.8). C-banding was used for constitutive heterochromatin localization.

Table 1 The effect of different REs on the autosomes, $X$ and B- chromosomes in E. plorans. Item 'type' groups similar bands obtained with different REs

\begin{tabular}{|c|c|c|c|c|c|}
\hline Type & Enzyme & $\begin{array}{l}\text { Restriction } \\
\text { site }\end{array}$ & Autosome & $X$ & B \\
\hline \multirow[t]{6}{*}{1} & Mbo I & GATC & + & + & + \\
\hline & Msp I & C CGG & + & + & + \\
\hline & Dde I & C TNAG & + & + & + \\
\hline & Scr FI & $\mathrm{CC} \quad \mathrm{NGG}$ & + & + & + \\
\hline & Sau 96A & G GNCC & + & + & + \\
\hline & Taq I & $\mathrm{T} \quad \mathrm{CGA}$ & + & + & + \\
\hline \multirow[t]{7}{*}{ II } & Sau $3 A$ & GATC & + & - & - \\
\hline & Hpa II & C CGG & + & - & - \\
\hline & Bam HI & C GATCC & + & - & - \\
\hline & Rsa I & GT $\quad \mathrm{AC}$ & + & - & - \\
\hline & Dpn I & GA $\quad \mathrm{TC}$ & + & - & - \\
\hline & Hind III & A AGCTT & + & - & - \\
\hline & Hinf I & G ANTC & + & - & - \\
\hline \multirow[t]{5}{*}{ III } & Hae III & GG $\quad \mathrm{CC}$ & + & - & + \\
\hline & Hin pI & $\mathrm{GC} \quad \mathrm{GC}$ & + & - & + \\
\hline & Pvu II & CAG CTG & + & - & + \\
\hline & Alu I & $\mathrm{AG} \quad \mathrm{CT}$ & + & - & + \\
\hline & Pst NI & CTGCA G & + & - & + \\
\hline IV & Hha I & GCG C & - & + & + \\
\hline V & Bst NI & $\mathrm{CC} \quad \mathrm{GG}$ & - & - & + \\
\hline \multirow[t]{3}{*}{ VI } & Ava II & G GWCC & - & - & - \\
\hline & Dra I & TTT AAA & - & - & - \\
\hline & Eco RI & G AATTC & - & - & - \\
\hline
\end{tabular}

(+) Presence of chromosome differentiation.

$(-)$ Absence of chromosome differentiation.

\section{Results}

\section{The chromosome complement}

Eyprepocnemis plorans is an orthopteran species with 23 acrocentric chromosomes in males (XO) and 24 in females $(\mathrm{XX})$. C-banding shows heterochromatic blocks in centromeric regions of all chromosomes (Fig. 1a). Populations of this species from the South-East of Spain show a complex polymorphism of B chromosomes (Henriques-Gil et al., 1982). The population of Eyprepocnemis plorans that we analysed had only one type of acrocentric B-chromosome which was homogeneously and deeply stained after C-banding. In only a few cases was a little differentiation between centromeric heterochromatin and euchromatin observed but this was not as marked as that obtained between autosomes or the X chromosome (Fig. 1a). B is stable in the germ line but its number may vary from one individual to another.

CMA3, a fluorescent DNA ligand which preferentially binds to GC-rich DNA (Ward et al., 1965; Berman et al., 1985), shows intense fluorescence in the centromeres of all chromosomes. However, the B-chromosome fluoresces homogeneously and more brightly than the centromeric blocks (Fig. 1b). Not all the heterochromatin in the centromeric blocks of the autosomes show CMA3 fluorescence. Those regions close to the end of the chromosomes, in close relationship with the kinetochores, are brighter. DAPI, a fluorescent DNA ligand which preferentially binds to AT-rich DNA (Kapuscinski \& Skoczylas, 1978), produces relatively uniform positive fluorescence in all chromosomes, including the B (Fig. 1c). The level of fluorescence in this B is lower than that obtained in the autosomes.

\section{Restriction endonucleases}

Results obtained with REs are described first with reference to their ability to produce linear differentiation in the autosomes, sex chromosomes and B-chromosomes. Using this criterion, results can be grouped into six classes (Table 1).

1 REs producing linear longitudinal differentiation in all chromosomes (Fig. 2a).

2 REs producing longitudinal differentiation in only the autosomes (Fig. 2b).

3. REs producing longitudinal differentiation in autosomes and B-chromosome but not affecting the X-chromosome (Fig. 2c).

4 REs producing longitudinal differentiation in the $B$ and the $\mathrm{X}$ chromosome (Fig. 2e).

5 REs only producing longitudinal differentiation in the B chromosome (Fig. 2d). 

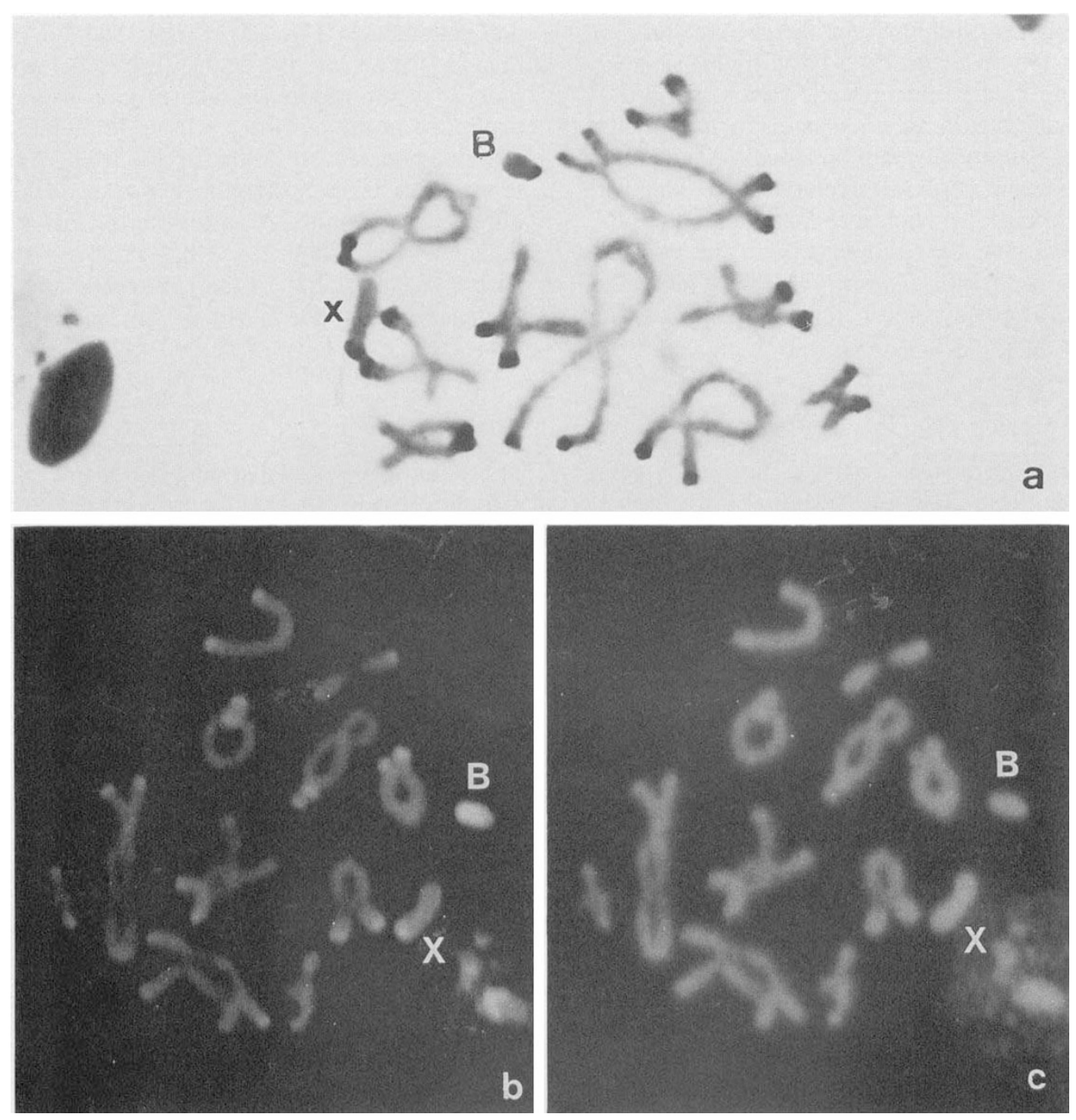

Fig. 1 Constitutive heterochromatin distribution in E. plorans with a B-chromosome. (a) C-banded diplotene. (b and c) Same diplotene stained with $\mathrm{CMA3}(\mathrm{b})$ and $\mathrm{DAPI}(\mathrm{c})$.

6 REs not producing linear differentiation in any chromosome.

The longitudinal differentiation produced in each case is enzyme-dependent, i.e. the bands produced by a given RE are not necessarily identical in size, chromosomal localization or staining characteristics to those produced by a different RE. Thus, for example, REs such as Mbo I or ScrF1 give rise to longitudinal differentiation in all chromosomes but the banding pattern obtained in each chromosome is different (compare Fig. 2a with $\mathrm{f}$ ). In order to give details on the abovelisted points we describe the results obtained with different REs on autosomes, sex and B-chromosomes.

\section{Banding patterns produced on the autosomes}

REs capable of producing clear-cut differentiation in autosomes can be subdivided into two categories: (i) those inducing a C-like banding pattern (Mbol, MspI, DdeI, BambHI, RsaI, HaelII, AluI, HinfI) (Fig. 2a; 3a); and (ii) those removing part of the constitutive heterochromatin (diminished $\mathrm{C}$-like bands), giving rise to small positively stained dots in each centromere region (Hin pI, ScrFI, Sau 96A, TaqI) (Fig. 2f; 3b). The heterochromatic regions from which material was removed stained less than undigested heterochromatin but more than euchromatin (Fig. 3b). This pattern was produced 
on all autosomes and also the $\mathrm{X}$ chromosome by certain REs. We thus refer to a basic model chromosome as being divided into three different regions: (i) heterochromatic region $h 1$, not cleaved by REs such as Hin pI, ScrFI, Sau 96A or TaqI and localized at the end of the chromosome, probably in close relationship with the kinetochores, (ii) heterochromatic region h2, cleaved by the same REs and adjacent to the previous one. The chromatin block composed of both h1 and h2 accounts for all constitutive heterochromatin of each chromosome, and (iii) euchromatin, E (Fig. 3c).

\section{Banding pattern produced on $X$ chromosome}

Fewer REs produced bands on the $\mathrm{X}$ chromosome (see Table 1). All these REs also produced a clear-cut differentiation on the autosomes, and the banding pattern obtained in this chromosome was similar to that produced on the autosomes. MboI, MspI and DdeI produced C-like banding in sex chromosomes (Fig. 3d) and autosomes (Fig. 2a). ScrFI, Sau 96A and TaqI digested part of the constitutive heterochromatic blocks (region $\mathrm{h} 2$ ) in a similar fashion to the digestion produced in the autosomes (Fig. 3e), while region h1 remained uncleaved. An exception to this banding pattern comes from Hhal, which produced longitudinal differentiation on the $\mathrm{X}$ and $\mathrm{B}$ but did not differentiate the autosomes (Fig. 2e). Hhal removed the heterochromatic block $\mathrm{h} 2$ on the centromere while leaving the euchromatic chromosome arm and the $\mathrm{h} 1$ region uncleaved (Fig. 3f).

\section{Banding pattern produced on the B-chromosome}

The heterogeneity of banding produced by digestion with different REs was more pronounced on the B than the $\mathrm{X}$ chromosome or autosomes. The results are classified as in Table 2 into (i) REs capable of revealing all centromeric heterochromatin (Fig. 4a) and (ii) REs capable of producing interstitial bands undetected by
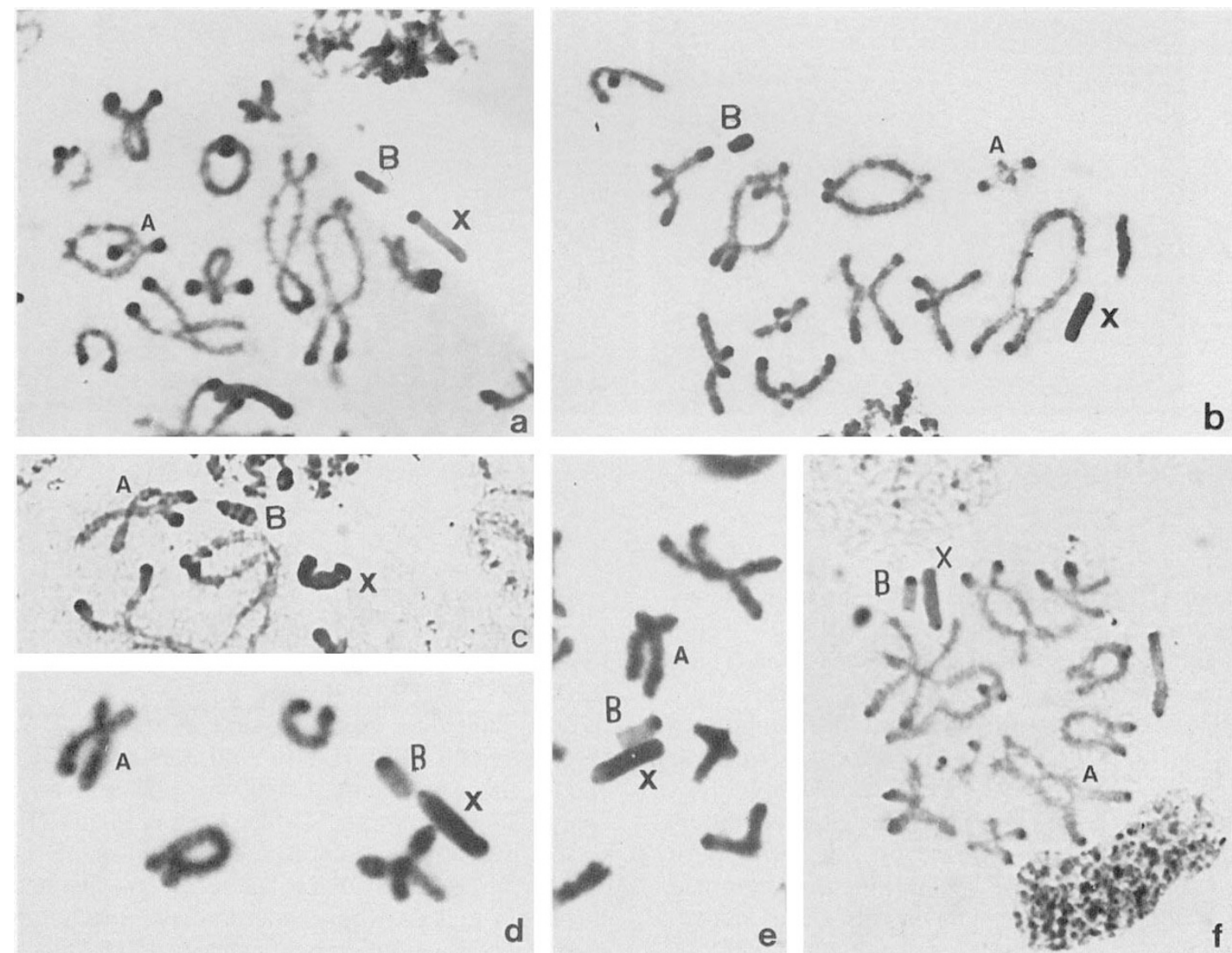

d

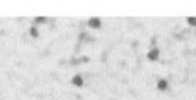

Fig. 2 Restriction nuclease banding in diplotene cells from individuals containing one B-chromosome. (a) Mbo I, (b) Bamb H1, (c) Alu I, (d) Bst NI, (e) Hha I, (f) Scr FI. 

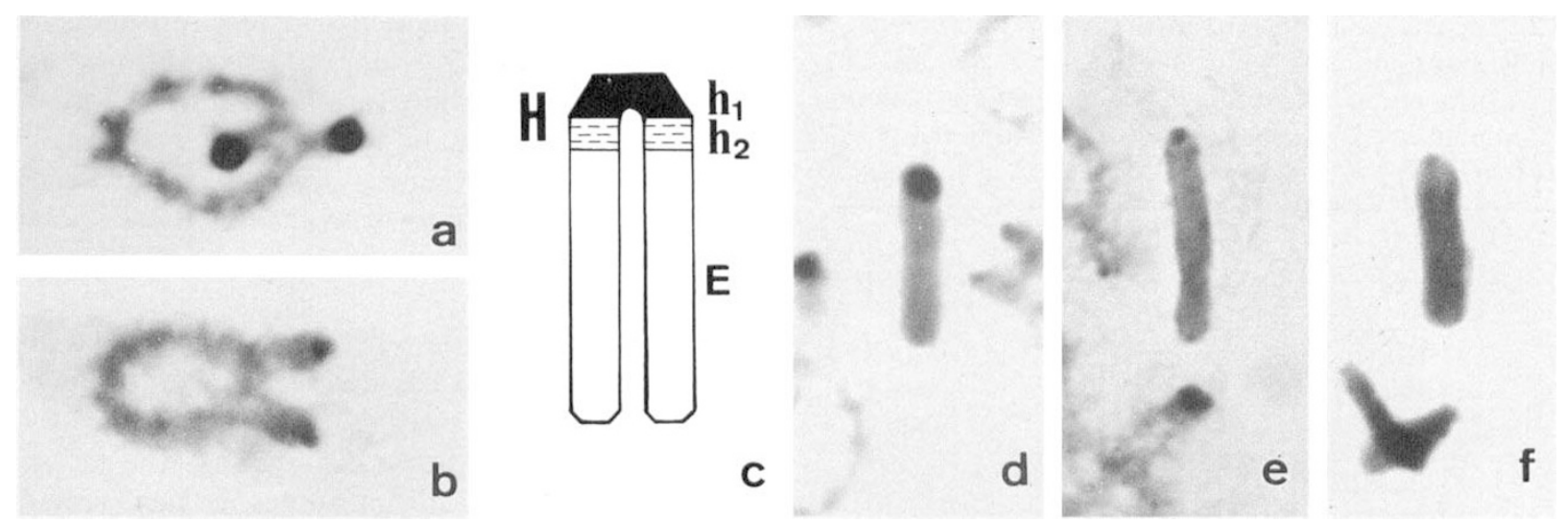

Fig. 3 Heterochromatin heterogeneity in E. plorans. Selected diplotene bivalents digested with Mbo I (a) and Scr FI (b). (c) A diagrammatic representation showing the distribution of different subsets of chromatin according to banding characteristics. (d-f) Selected X-chromosomes showing differential digestibility of chromatin: Mbo I (d), Scr FI (e) and Hha I (f).

other banding methods (Fig. 4b). In the latter case a centromeric plus two interstitial bands were obtained. The level of differentiation obtained in these interstitial bands varied from one RE to another. Thus, for example, while AluI left both bands uncleaved (Fig. $2 \mathrm{c}$ ), MboI produced more intense digestion of the interstitial band near the centromere (Fig. 4b). When the B-chromosome was digested with MspI additional differences in these interstitial bands were detected depending on the incubation time. Short digestion $(2 \mathrm{~h})$ with this enzyme produced the two interstitial bands mentioned above (Fig. 4c). At increasing digestion times $(7-8 \mathrm{~h})$, the band closest to the centromere tended to lose stainability (Fig. 4d). Longer digestions (overnight) produced massive extraction of material from both bands and only the centromere remained stained. This effect confirms the existence of the structural differences between these bands observed with REs such as Mbol.

Two points should be emphasized: (i) Hin pI, ScrFI, Sau 96A and TaqI, REs which remove part of the constitutive heterochromatin ( $\mathrm{h} 2$ region) in autosomes and in the $\mathrm{X}$ chromosome, do not digest the centromeric constitutive heterochromatin in the B-chromosome; (ii) additional interstitial bands are produced on the B-chromosome with REs such as Mbol, MspI, DdeI, PvuII, AluI, Pst NI, HinfI and HaelII; most of these REs give rise to paracentromeric bands on the autosomes. This fact could be of importance in understanding the origin of this extra chromosome as will be discussed later.

\section{Digestion with non-specific nucleases}

Incubation of chromosomes with $\mathrm{S} 1$ nuclease produced a pronounced C-like banding pattern in auto- somes and in the $\mathrm{X}$ chromosome. In the B-chromosome, it was possible to observe a centromeric band as well as the two additional interstitial bands that were also revealed by some REs (Fig. 4e). In all cases, S1 digestion produced a dramatic loss of euchromatin in the chromosome arms and gave rise to high contrast between eu- and heterochromatin after staining (Fig. 4f).

Bal 31 and ExolII do not induce any different staining in any chromosome; they do not remove DNA differentially from eu- and heterochromatin.

\section{Discussion}

In purified DNA REs act by cleaving specific base sequence targets and they also show the capability to act on the DNA of fixed chromosomes (Mezzanotte $e t$ al., 1983; Miller et al., 1983). The extraction of DNA from fixed chromosomes by REs can be affected by a series of factors such as fixation of the material (Burkholder, 1989), cleavage frequency (Miller et al., 1983; 1984; Bianchi et al., 1985; Mezzanotte, 1986) or structure of the RE and chromatin (Gosálvez et al., 1989). Even so we believe that under homogeneous experimental conditions REs may be used to study processes that determine pathways of evolution of a given karyotype through the sensitivity of each chromosome region to a given RE. In this context, the results obtained in the present experiment, summarized in Table 2, indicate the similarities and differences that underlie the genome of this species. Thus, while the action of each RE on the autosomes produces a pattern that is both homologous and conservative, differences exist among autosomes, sex chromosome and Bs. This clearly points to a different evolution pattern for each type of chromosome. 
Table 2 The common effects (compare boxes) of different REs in B-, autosomes and X-chromosomes in E. plorans. REs have been plotted according to a $\mathrm{C}$-like banding pattern (item 1), diminished C-like bands (item 2) or additional bands (item 3)

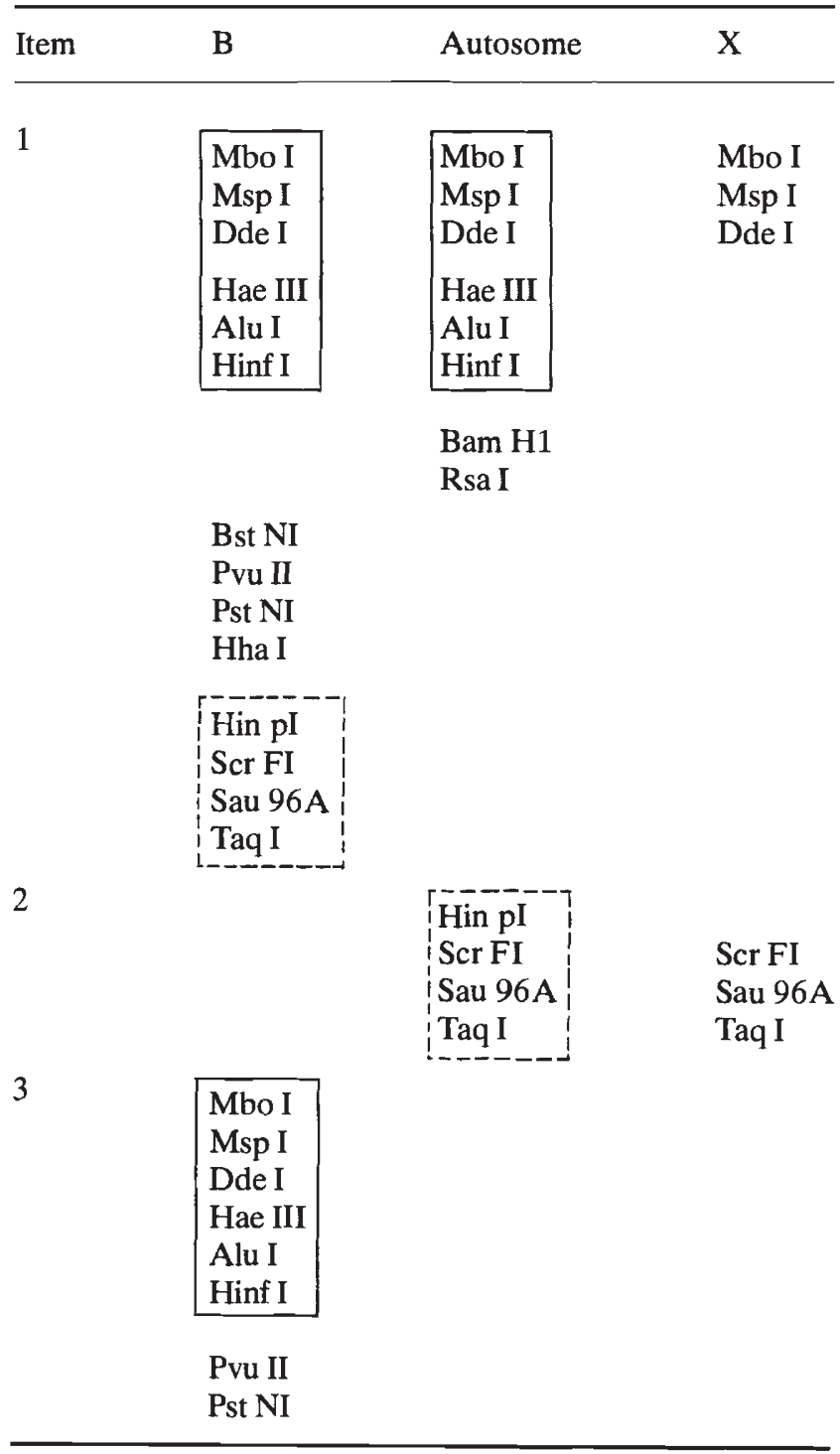

Interesting differences are also revealed by differential action of some REs within the heterochromatin. The existence of two identifiable classes of chromatin (h1/h2) within homogeneous C-bands, similarly distributed through each autosome, agrees completely with the principle of equilocal distribution of heterochromatin (Schweizer \& Loidl, 1987). In support of this principle, which seems to be widespread in Orthoptera, we may recall the similar response of all centromeric heterochromatin observed in the chromosomes of the grasshopper Oedipoda germanica after insitu cleavage with different REs (López-Fernández et al., 1989) or the homogeneous distribution of a parti- cular repeated DNA sequence in all centromeric heterochromatin of the grasshopper Atractomorpha similis. These repeated families are commonly confined to specific heterochromatic regions, either proximally or distally (John et al., 1986). Observations presented in this paper with the fluorochromes or those shown by John et al. (1985), using the same dyes, also support this idea.

It appears that the distribution of the different classes of chromatin has diverged in the evolution of the genome of this species. This divergence is clear when comparing the heterochromatin of the autosomes with that of the sex chromosomes. In fact, only three REs (MboI, MspI, DdeI) produce the same banding pattern in autosomes and X chromosome: all remaining REs induce clear-cut bands in autosomes, while being incapable of affecting the $\mathrm{X}$ chromosome (Table 2). Despite the divergence that occurred among the different subsets of chromatin, some similarities were observed between the autosomal heterochromatin and that of the B chromosome, but this has different implications from those between autosomes and sex chromosomes. Thus, only two REs (Bamb HI and $\mathrm{RsaI}$ ) among those that produced C-like bands in the autosomes did not induce $\mathrm{C}$-like bands in the $\mathrm{B}$ chromosome. Six REs that were able to produce C-like bands in the autosomes also gave rise to C-like bands in the B-chromosome, and produced, in addition, interstitial bands in the B-chromosome. The same result was also produced by PvuII and Pst NI (Table 2). In addition, four REs (Hin pI, Scr FI, Sau 96A and Taq I) gave rise to $\mathrm{C}$-like bands on the $\mathrm{B}$ but reduced C-bands on the autosomes (Table 2).

The origin of the $\mathrm{B}$ chromosomes is uncertain. They do not pair with segments of the A chromosome complement, size and morphology do not correspond to other members of the complement and we lack information about other characteristics such as the composition and distribution of distinctive satellite DNAs (see Jones \& Rees, 1982 for review). Various origins have been suggested for B-chromosomes. For example, Hewitt (1979) suggested that X chromosomes and polysomic chromosomes, which undergo a suppression of the deleterious effects of repressing their regular activity, may persist stochastically in a population. These elements are potentially B-chromosomes. The fact that in some well-studied cases it has been possible to demonstrate the existence of transcription involving nucleolar organizer regions (Cabrero et al., 1987; Maluszynska \& Schweizer, 1989) indicates an ancestral relationship with the A chromosomes. The results obtained in E. plorans also suggest this ancestral resemblance. Note that, as stated above, the action of certain REs shows structural simi- 


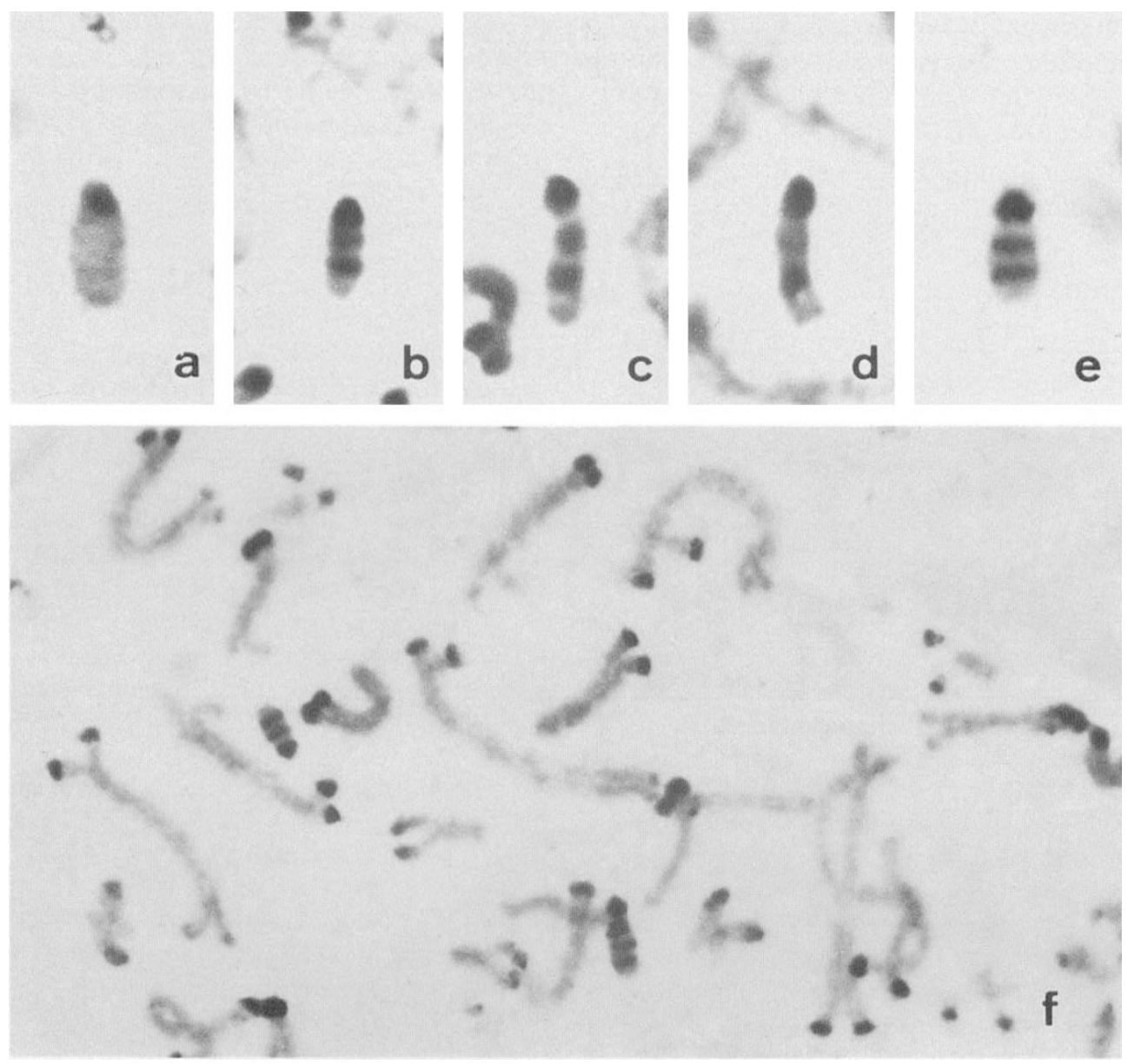

Fig. 4 Selected B-chromosomes in E. plorans after digestion with Bst NI (a), Mbo I (b). Msp I after 2 h digestion (c) or overnight (d) and S1 nuclease (e).(f) Early diplotene digested with S1.
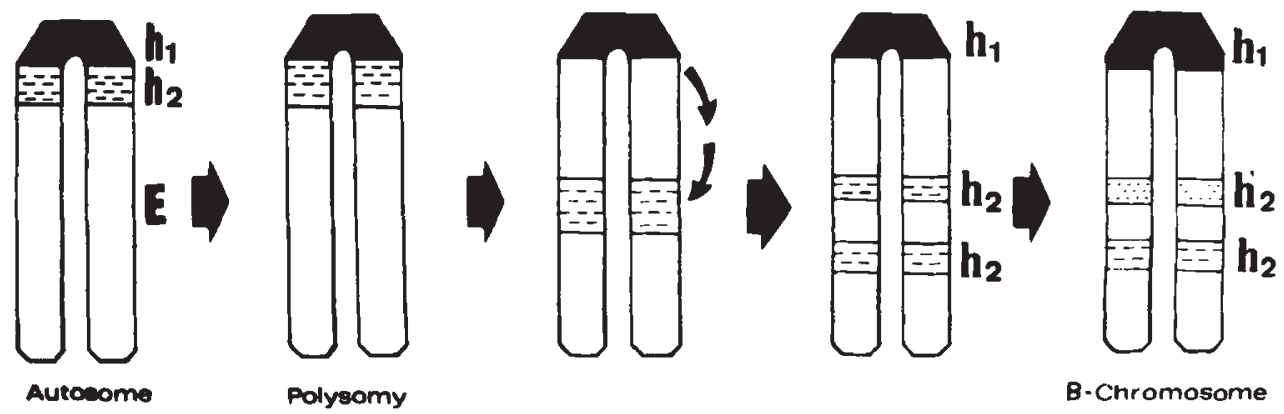

Fig. 5 Diagrammatic representation of the possible origin of the B-chromosome in E. plorans from a centric autosome fraction involving different chromosome rearrangements.

larity between specific regions, i.e. constitutive heterochromatin of autosomes and B-chromosome (see information summarized in Table 2). This leads us to hypothesize that the B-chromosome is derived through a series of events from an autosome. In particular, we postulate that the following steps have occurred: (i) production of polysomy; (ii) chromosome rearrange- ment (inversion?) involving heterochromatic region h2 and part of eu-chromatic (E) and (iii) amplification of the proximal $h 1$ region and reorganization of region $h 2$ to give rise to an incipient differentiation between bands $\mathrm{h} 2$ and $\mathrm{h}^{\prime} 2$ (Fig. 5 ).

In conclusion, our data indicate that evolutionary processes in the E. plorans karyotype have produced 
some degree of heterogeneity within different fractions of chromatin, as in other cases studied. They also allow us to postulate that the material that forms this B-chromosome possibly arises from a number of evolutive steps involving amplification and reorganization of autosomal material.

\section{Acknowledgements}

We would like to acknowledge Professor G. M. Hewitt and M. A. Groeneweg for suggestions and discussion. This work was partially supported by grant SC000127 from EEC.

\section{References}

BERMAN, E., BROWN, S. C., JAMES, T. L. AND SHAFER, R. H. 1985. NMR studies of chromomycin A3 interaction with DNA. Biochemistry, 24, 6887-6893.

BIANCHI, M. S., BIANCHI, N. O., PANTELIAS, G. E. AND WOLFF, S. 1985. The mechanism and pattern of banding induced by restriction endonucleases in human chromosomes. Chromosoma, 91, 131-136.

BURKHOLDER, G. 1989. Morphological and biochemical effects of endonucleases on isolated mammalian chromosomes in vitro. Chromosoma, 97, 347-355.

CABRERO, J., ALCHE, J. D. AND CAMACHO, J. P. M. 1987. Effects of B chromosomes on the activity of nucleolar organizer regions in the grasshopper Eyprepocnemis plorans: activation of a latent nucleolar organizer region on a $\mathrm{B}$ chromosome fused to an autosome. Genome, 29, 116-121.

GOSÁLVEZ, J., BELLA, J. L., LOPEZ-FERNÁNDEZ, C. AND MEZZANOTTE, R. 1987. Correlation between constitutive heterochromatin and restriction enzyme resistant chromatin in Arcyptera tornosi. Heredity, 59, 173-180.

GOSÁLVEZ, J., LOPEZ-FERNÁNDEZ, C., FERRUCCI, L. AND MEZZANOTTE, R. 1989. DNA base sequence is not the only factor accounting for restriction endonucleases activity on metaphase chromosomes: evidence using isoschizomeres. Cytogenet. Cell Genet., 50, 142-144.

HENRIQUES-GIL, N., SANTOS, J. L. AND GIRALDEZ, R. 1982. B-chromosome polymorphism and interchromosomal chiasma interference in Eyprepocnemis plorans (Acrididae: Orthoptera). Chromosoma, 85, 349-359.

HEwIT, G. M. 1979. Animal Cytogenetics. 3 Insecta 1. Orthoptera. Gebruder Borntraeger, Berlin.

JOHN, B., APPELS, R. AND CONTRERAS, N. 1986. Population cytogenetics of Atractomorpha similis II Molecular character- isation of the distal C-band polymorphisms. Chromosoma, 94, 45-58.

JOHN, B., KING, M., SCHWEIZER, D. AND MENDELAK, M. 1985. Equilocality of heterochromatin distribution and heterochromatic heterogeneity in acridid grasshoppers. Chromosoma, 91, 185-200.

JONES, R. N. AND REES, H. 1982. B Chromosomes. Academic Press, London.

KAPUSCINSKI, J. AND SKOCZYLAS, B. 1978. Fluorescent complexes of DNA with DAPI 4',6-diamidine-2-phenyl indole. $2 \mathrm{HCl}$ or $\mathrm{DCl} 4^{\prime}, 6$-dicarboxyamide-2-phenyl indole. Nuc. Acid Res, . 5, 3775-3799.

LÓPEZ-FERNÁNDEZ, C., GOSÁLVEZ, J. AND MEZZANOTTE, R. 1989. Heterochromatin heterogeneity in Oedipoda germanica (Orthoptera) detected by in situ digestion with restriction endonucleases. Heredity, 62, 269-277.

MALUSZYNSKA, J. AND SCHWEIZER, D. 1989. Ribosomal RNA genes in B chromosomes of Crepis capillaris detected by non-radioactive in situ hybridization. Heredity, 62, 59-66.

MEZZANOTTE, R. 1986. The selective digestion of polytene and mitotic chromosomes of Drosophila melanogaster by Alu I and Hae III restriction endonucleases. Chromosoma, 93, 249-255.

MEZZANOTTE, R., FERRUCCI, L., VANNI, R. AND BIANCHI, U. 1983. Selective digestion of human metaphase chromosomes by Alu I restriction endonuclease. J. Histochem. Cytochem., 31, 553-556.

MILLER, D. A., CHOI, Y. AND MILLER, O. J. 1983. Chromosome localization of highly repetitive human DNA's and amplified ribosomal DNA with restriction enzymes. Science, 219, 395-397.

MILleR, D. A., GOSDEN, J. R., HASTIE, N. D. AND EVANS, H. J. 1984. Mechanism of endonuclease banding of chromosomes. Exp. Cell Res., 155, 294-298.

SCHWEIZER, D. 1976. Reverse fluorescent chromosome banding with chromomycin and DAPI. Chromosoma, 58, 307-324.

SCHWEIZER, D. 1980. Simultaneous fluorescent staining of $\mathrm{R}$ bands and specific heterochromatic regions (DADAPI) bands in human chromosomes, 27, 190-193.

SCHWEIZER, D. AND LOIDL, J. 1987. A model for heterochromatin dispersion and the evolution of C-bands patterns. Chromosome Today, 9, 61-79.

SUMNER, A. T. 1972. A simple technique for demonstrating centromeric heterochromatin. Exp. Cell Res., 75, 304-306.

WARD, D. C., REICH, R. AND GOLDBERG, 1. M. 1965. Base specificity in the interaction of polynucleotides with antibiotic drugs. Science, 149, 1259-1263. 